\title{
Equilíbrio e marcha de idosos residentes em instituições asilares do município de Catanduva, SP
}

\section{Balance and gait of elderly people at retirement homes in Catanduva city, SP, Brazil}

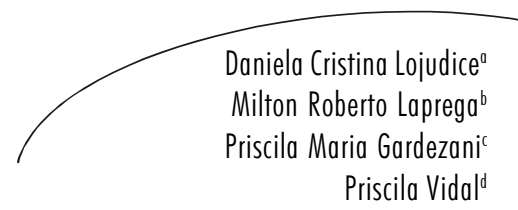

\section{Resumo}

O presente trabalho tem como objetivos avaliar o equilíbrio e marcha de idosos institucionalizados e caracterizar os idosos que se apresentaram com alterações no equilíbrio e marcha segundo sexo, faixa etánia, estados visual e auditivo. Este estudo, do tipo descritivo e de caráter transversal, foi realizado nas instituições asilares do município de Catanduva (SP), constituído de idosos de ambos os sexos. Os idosos foram responderam primeiramente a um questionánio que trazia informações sobre identificação e condições de saúde. O equilíbrio e marcha foram avaliados através da Escala de Equilíbrio e Marcha de Tinetti. Foram avaliados 105 idosos, sendo 62 (59\%) do sexo feminino e 43 (41\%) do sexo masculino. A idade variou de 60 a 97 anos, com média de 79,2 + (9,7) anos para o sexo feminino e 73,2 + $(9,3)$ anos para o sexo masculino. Houve associação entre dificuldade no equilíbrio e marcha e sexo feminino $(p=0,003)$; faixa etána $(p=0,004)$ e dificuldade na visão $(p=0,008)$. Não houve diferença entre dificuldade no equilíbrio e marcha e deficiência auditiva. De acordo com os resultados, concluiu-se que entre os idosos residentes em asilos da cidade de Catanduva, os distúrbios do equilíbrio e marcha estão associados à idade avançada, sexo feminino e à deficiência visual.

\section{Abstract}

This paper aims to evaluate balance and gait in elderly people who live at retirement homes and to characterize alterations in balance and walk according to sex, age,

\author{
Palavras-chave: \\ idoso; estudos de \\ avaliação; equilí- \\ brio musculos- \\ quelético; marcha; \\ saúde do idoso \\ institucionalizado; \\ instituição de longa \\ permanência para \\ idosos; epide- \\ miologia descritiva; \\ estudos transver- \\ sais; escalas; \\ Catanduva (SP)
}

Universidade de São Paulo

Faculdade de Medicina, Ciências Médicas

Ribeirão Preto, SP, Brasil

Correspondência / Comespondenœ

Daniela Cristina Lojudice

Rua Corumbá, 411

15804-345 - Catanduva, SP, Brasil

E-mail: daniela_lojudice@hotmail.com 
vision and hearing. The research, of descriptive type and transversal character, was conducted at Catanduva city's retirement homes, with elderly people of both sexes. Firstly, the elderly answered a questionnaire with information about identification and health conditions. Balance and gait were evaluated through Tinetti Balance and Gait Scales. One hundred and five elders were evaluated, being 62 (59\%) women and 43 (41\%) men, aged between 60 to 97 years, being 79,2 $\pm 9,7$ years to females and 73,2 $\pm 9,3$ years to males. There was association between balance and gait's difficulty and female sex $(p=0,003)$; age $(p=0,004)$ and vision's difficulty $(p=0,008)$. There was no difference between balance and gait and heaning disability. According to the results, the conclusion is that in Catanduva city's retirement homes balance and gait disability are associated with age, female sex and vision disability.

\section{Key words aged;}

evaluation studies; musculoskeletal equilibrium; gait; health of institutionalized elderly; homes for the aged; epidemiology descriptive; crosssectional studies; scales; Catanduva city (SP)

\section{INTRODUÇÃO}

A população idosa vem crescendo desde o início da década de 1960 em decomência do aumento da taxa de fecundidade e da redução da taxa de mortalidade levando, portanto, ao estreitamento da base da pirâmide populacional $1^{1,2}$

Dados do Censo de 2000 apontam que pessoas com idade acima de 60 anos representam 8,56\% da população brasileira, dos quais $55 \%$ são mulheres. A expectativa de vida da população brasileira foi estimada em 68 anos e 7 meses. $^{3}$

De acordo com Freitas etal, ${ }^{2}$ o envelhecimento se define como a manifestação de eventos biológicos que ocorrem ao longo de um peńodo, em ritmos diferentes para cada pessoa. Representa as perdas na função nomal que continuam até a longevidade máxima Papaléo Netto ${ }^{4}$ conceitua o envelhecimento como um processo dinâmico e progressivo, no qual há modificações morfológicas, funcionais, bioquímicas e psicológicas que determinam a perda da capacidade de adaptação do indivíduo ao meio ambiente, causando maiorvulnerabilidade e maiorincidência de processos patológicos que terminam porlevá-lo à morte.

À medida que o indivíduo envelhece, ocorrem alterações nos seus sistemas em geral, levando ao aparecimento de doenças crônicas, declínio da capacidade funcional, perda da autonomia e independência. ${ }^{5-8}$

De acordo com Baloh etal, ${ }^{9}$ Boers etal. ${ }^{5} \mathrm{e}$ Steadman etal, ${ }^{10} 0$ equilíbrio é um dos sistemas mais afetados com o envelhecimento, 0 que propicia o surgimento de instabilidadese a ocorrência de quedas entre idosos. Esses autores ainda relatam que as quedas irão resultar em consideráveis custos de serviços sociais e da saúde, devido às lesões, maior número de intemações, bem como uma maiorperda de confiança e dependência nas ati- 
vidades básicas funcionais.

A marcha também se altera com o envelhecimento, em decorrência do acometimento de vánios sistemas e órgãos responsáveis pelo controle motor. Dessa forma, o idoso fica mais susceptível a tropeçare a cair. ${ }^{9,11,12,13}$

Um dos testes mais utilizados com o objetivo de venificar os padrões de mobilidade e determinar a possibilidade da ocomência de quedas entre idosos é o "Performance Oniented Mobility Assessment" (POMA). ${ }^{8}$ Esse instrumento avalia o equilibrio do idoso em diversas posições, além de avaliar a marcha. É um método confiável e sensível às mudanças significativas. ${ }^{14}$

Embora já se tenha conhecimento de que os estados de equilíbrio e marcha são afetados com o envelhecimento, pouco se sabe das reais dificuldades nesses sistemas em idosos institucionalizados, muitas vezes considerados mais fragilizados e com maior dependência funcional. Diante disso, surgiu o interesse pelo presente trabalho, que tem como objetivos avaliar os estados de equilíbrio e marcha de idosos residentes em instituições asilares e caracterizaridosos que se apresentaram com alterações no equilíbrio e marcha segundo sexo, faixa etánia, estados visual e auditivo.

\section{CASUISTIICA E MÉTODOS}

Trata-se de estudo descritivo, de caráter transversal, realizado no peńodo de agosto a dezembro de 2004, nas instituições asilares do município de Catanduva, estado de São Paulo.

A população do estudo foi constituída por todos os idosos ( 60 anos ou mais), de ambos os sexos, residentes nas instituições asilares do município. Os idosos tiveram que se apresentarconscientes e onientados no tempo e no espaco, capazes de interagirem em uma entrevista e estar institucionalizados por um peníodo mínimo de seis meses. Todos os participantes assinaramo Termo de Consentimento Livree Esclarecido, segundo a Resolução no 196/96 do Conselho Nacional de Saúde..$^{15}$ Foramexcluídos osidosos que se recusaram a participar do estudo, bem como os que não deambulavam e aqueles com idade inferior a 60 anos.

Para a coleta de dados, utilizou-se entrevista com um questionánio com informações referentes a sexo, faixa etánia, estados da visão e audição, prática de atividade física (peníodo mínimo de três vezes semanais) e ocomência de quedas nos últimos seis meses. Em seguida foi aplicada a Escala "Performance - Oriented Mobility Assessment" (POMA), ${ }^{8}$ que busca informações sobre equilíbrio sentado, levantandose, em pé e sentando; tentativas de se levantar, início da marcha; comprimento e altura dos passos; direção; estabilidade do tronco ao desenvolvero percurso e distância dos tornozelos. ${ }^{2}$ Essa escala apresenta pontuação máxima igual a 28, sendo que o escore menor que 19 indica o nisco cinco vezes maior 
de quedas. ${ }^{8}$ De acordo com Whitney etal., ${ }^{14} \mathrm{a}$ escala apresenta confiabilidade interativa adequada e validade de conteúdo.

Análise estatística dos dados: a análise descritiva foi apresentada na forma de média, desvio padrão, valores mínimos e máximos para as vanáveis numéncas. Para investigara associação entre baixo desempenho na escala de Tinetti - POMA (escore infeniora 19) esexo, faixa etána e estados de visão e audição, prática de atividade física e ocomência de quedas, foram utilizados os testes do Qui-quadrado e exato de Fisher. O nível de significância foi estipulado em $5 \%$.

O presente trabalho foi aprovado pelo Comitê de Ética do Centro de Saúde Escola de Ribeirão Preto, SP.

\section{RESULTADOS}

A população estudada foi composta de
105 idosos. Desses, 62 (59\%) eram do sexo feminino. A idade dos idosos variou de 60 a 97 anos, com média de 79,2 \pm 9,7 anos para o sexo feminino e 73,2 $\pm 9,3$ anos para o masculino.

Do total dos participantes do estudo, 52 (49,5\%) apresentaram alterações no equilíbrio e marcha, ou seja, apresentaram pontuação menor que 19 na escala de Tinetti (POMA), indicando maior nisco de quedas nessa população.

Verificou-se, também, que dos 52 idosos que se apresentaram com baixo desempenho no POMA, 36 (69,3\%) eram do sexo feminino. Houve diferença estatística significante entre baixo desempenho no POMA e sexo feminino $(p=0,0036$; tabela 1$)$.

No que tange à faixa etánia dos idosos, dos 52 idosos que apresentaram escore infenor a 19 na escala de Tinetti, 10 (19,2\%) ti-

Tabela 1 Distribuição dos escores da Escala "Performance-Oriented Mobility Assessment" (POMA) na população idosa institucionalizada, segundo sexo, 2004.

\begin{tabular}{lccccrr}
\hline $\begin{array}{c}\text { Escores no } \\
\text { POMA }\end{array}$ & Feminino $\%$ & Masculino & $\%$ & & \multicolumn{2}{c}{ Total } \\
\hline Escore $<19$ & 36 & $69,3 \%$ & 16 & $30,7 \%$ & 52 & $49,5 \%$ \\
Escore $\geq 19$ & 26 & $49,0 \%$ & 27 & $51,0 \%$ & 53 & $50,5 \%$ \\
Total & 62 & $59,0 \%$ & 43 & $41,0 \%$ & 105 & $100 \%$ \\
\hline
\end{tabular}

nham idade entre 60 e 69 anos, 14 (26,9\%), entre 70 e 79 anos, e 28 (53,9\%) tinham idade igual ou supeniora 80 anos. Percebeu-se, portanto, que escores baixos na escala de avaliação de equilíbrio e marcha (POMA) são mais freqüentes entre pessoas mais velhas $(p=0,004$; tabela 2).

Quanto ao estado visual dos entrevistados, dos 52 idosos que se apresentaram com baixo desempenho na escala, 18 (34,6\%) relataram 
Tabela 2- Distribuição dos escores da Escala "Performance-Oriented Mobility Assessment" (POMA) na população idosa institucionalizada, segundo faixa etária, 2004.

\begin{tabular}{lccccccccc}
\hline \multicolumn{1}{c}{\begin{tabular}{c} 
Escores no \\
\multicolumn{1}{c}{ POMA }
\end{tabular}} & $<70$ anos & $\%$ & \multicolumn{2}{c}{ Faixa Etária } \\
\hline Escore $<19$ & 10 & $19,2 \%$ & 14 & $26,9 \%$ & 28 & $53,9 \%$ & 52 & $49,5 \%$ \\
Escore $\geq 19$ & 17 & $32,0 \%$ & 24 & $45,3 \%$ & 12 & $22,7 \%$ & 53 & $50,5 \%$ \\
Total & 27 & $25,7 \%$ & 38 & $36,2 \%$ & 40 & $38,1 \%$ & 105 & $100 \%$ \\
\hline
\end{tabular}

ter visão nuim, 21 (40,4\%) ter visão regular e somente 13(25\%) relataram terboavisão. Houve diferença estatisticamente significante entre baixo desempenho no POMA e dificuldade vi- sual $(p=0,008$; tabela 3$)$.

No que se refere ao estado auditivo, dos 52 idosos que se apresentaram com dificuldades no equilíbrio e marcha, $32(61,6 \%)$

Tabela 3 - Distribuição dos escores da Escala “Performance-Oriented Mobility Assessment” (POMA) na população idosa institucionalizada, segundo estado visual, 2004.

\begin{tabular}{llccccccc}
\hline \multicolumn{1}{c}{ Escores no } & \multicolumn{9}{c}{ Estado Visual } & \multicolumn{3}{c}{ Total } \\
\multicolumn{1}{c}{ POMA } & Bom & $\%$ & Regular & $\%$ & Ruim & & \\
\hline Escore $<19$ & 13 & $25,0 \%$ & 21 & $40,4 \%$ & 18 & $34,6 \%$ & 52 & $49,5 \%$ \\
Escore $\geq 19$ & 28 & $52,8 \%$ & 17 & $32,1 \%$ & 8 & $15,1 \%$ & 53 & $50,5 \%$ \\
Total & 41 & $39,0 \%$ & 38 & $36,2 \%$ & 26 & $24,8 \%$ & 105 & $100 \%$ \\
\hline
\end{tabular}

relataram terboa audição, 16 (30,8\%), teraudição regulare apenas $4(7,6 \%)$ relataram ter audição nuim. Não houve diferença estatisticamente significante entre dificuldade auditiva e baixo desempenho na escala de Tinetti.

Ao realizaro teste do Qui-quadrado para venificar a associação entre baixo desempenho no POMA e prática de atividade física, venificou-se que não houve associação significante entre eles $(p=0,073)$. Apesar de não ter havido relação entre essas variáveis, constatou-se, no presente estudo, que dos 52 idosos que apresentaram baixo desempenho no POMA, 39 (75\%) não exerciam atividade física, ou seja, a dificuldade no equilíbrio e marcha foi mais freqüente entre aqueles idosos que não a praticavam.

Quanto à ocomência de quedas, pode-se concluirque as quedas foram mais freqüentes $(61,9 \%)$ entre osidosos que se apresentaram com baixo desempenho no POMA ( $p=0,038$; tabela 4).

\section{DISCUSSÃO}


Tabela 4- Distribuição dos escores da Escala "Performance-Oriented Mobility Assessment" (POMA) na população idosa institucionalizada, segundo ocomência de quedas, 2004.

\begin{tabular}{|c|c|c|c|c|c|c|}
\hline \multirow{2}{*}{$\begin{array}{c}\text { Escores no } \\
\text { POMA }\end{array}$} & \multicolumn{4}{|c|}{ Ocorrência de Quedas } & \multirow{2}{*}{\multicolumn{2}{|c|}{ Total }} \\
\hline & $\operatorname{Sim}$ & $\%$ & Não & $\%$ & & \\
\hline Escore $<19$ & 26 & $61,9 \%$ & 26 & $41,3 \%$ & 52 & $49,5 \%$ \\
\hline Escore $\geq 19$ & 16 & $38,1 \%$ & 37 & $58,7 \%$ & 53 & $50,5 \%$ \\
\hline Total & 42 & $40,0 \%$ & 63 & $60,0 \%$ & 105 & $100 \%$ \\
\hline
\end{tabular}

Podemos verificar, no presente trabalho, que a dificuldade no equilibrio e marcha em idosos institucionalizadas, representada pelo baixo desempenho na escala de Tinetti, foi bastante freqüente, pois atingiu a metade da amostra estudada.

De acordo com Tinetti, ${ }^{10}$ Tinetti etal., ${ }^{13}$ Whitney etal., ${ }^{14}$ Baloh et al., ${ }^{9}$ Rosa etal. ${ }^{7} \mathrm{e}$ Rodrigues etal., ${ }^{6}$ as dificuldades de equilíbrio e marcha são freqüentes entre idosos, pois no decorrer do processo de envelhecimento, as disfunções do aparelho locomotor e do sistema sensorial são presentes e contribuem para instabilidade e ocorrência de quedas nessa população.

Os dados da presente investigacãa, referentes ao menor desempenho na escala de Tinetti ser maior no sexo feminino, não foram diferentes do estudo de Rodrigues \& al. ${ }^{6}$ Esses autores avaliaram os estados de equilibnio e marcha de 51 idosos residentes em asilos e verificaram que as mulheres apresentam maior déficit de equilibrio e marcha em relação aos homens. As mulheres, aém de se apresentarem com menor massa musculare conseqüente diminuição da força muscular quando comparadas aos homens, apresentam maiornúmero de doenças crônicas, o que provavelmente contribui para uma menorcapacidade funcional nessa população.

Pode-se concluir, no presente estudo, que o déficitde equilíbrio e marcha foi maior entre os idosos mais velhos. É sabido que, no decorrerdo envelhecimento, os idosos adotam mecanismos compensatónios para mantera postura adequada e uma marcha funcional, tais como: alargamento da base de suporte, diminuição do comprimento e altura do passo e a redução da velocidade da marcha. Dessa forma, o idoso está mais susceptível a tropeçar e a cair.

Evidenciou-se, no presente estudo, que houve associação significante entre baixo desempenho na escala de Tinetti e o estado visual, apurando-se que os idosos com menordesempenho na escala foram aqueles que relataram ter dificuldade visual. Para Freitas etal., ${ }^{2}$ o sistema visual é o mais importante sistema de informação sensonial, o qual envia informações ao Sistema Nervoso Central e detecta os deslocamentos posturais sobre a posição e os movimentos do como em relação ao ambiente externo. A detenioração da perspicácia visual, bem como a dimi- 
nuição da velocidade de adaptação ao escuro e o aumento do limiar de percepção luminosa, predispõe o idoso a um déficit de equilíbrio e marcha. ${ }^{6,9,11-13}$

Apesarde não ter havido relação positiva entre dificuldade auditiva e deficiência no equilíbrio e marcha no presente estudo, a literatura aponta que a diminuição da sensibilidade auditiva contribui para as disfunções do equilíbrio e da locomoção e, como conseqüência, há maior probabilidade da ocomência de quedas. ${ }^{6,89,13}$ Desta maneira, exames periódicos com a finalidade de avaliar os estados de visão e audição devem serfrequentemente realizados, com o intuito de minimizaras chances de ocorrência de quedas.

Quanto à prática de atividade física, apesar de não ter havido associação significante entre ausência de atividade física e baixo desempenho no POMA, verificou-se, na presente investigação, que a maionia dos idosos com baixo desempenho na escala de equilibrio e marcha não exercia atividade física. No estudo de Steadman etal., ${ }^{10}$ foi comprovado que idosos que realizavam atividades físicas, através de terapias convencionaise avançadas, apresentaram melhonias nos estados do equilíbrio e da marcha, com conseqüente diminuição do número de quedas. Segundo Boers etal., ${ }^{5}$ a atividade física irá prevenir ou minimizar as mudanças decorrentes do processo de envelhecimento, reduzindo a incidência e a gravidade das quedas. Dessa maneira, exercícios físicos devem serenfatizados, pois beneficiam a saúde e melhoram a capacidade funcional.

Evidenciou-se, no presente estudo, quehou- ve associação significante entre baixo desempenho na escala de Tinetti e a ocomência de quedas, apurando-se que as quedasforam mais freqüentes entre os idosos com menordesempenho na escala. Resultados semelhantes foram encontrados nos estudos de Pereira etal. ${ }^{12} \mathrm{e}$ Baloh etal ${ }^{9}$. As quedas são eventos importantes na vida de idosos e interferem, de maneira negativa, na qualidade de vida dosmesmos. Diante disso, o reconhecimento dos idosos com maiores dificuldades para se manterem postura ortostática e para caminhar, bem como o incentivo à prática de fisioterapia, visando ao fortalecimento musculare ganho de amplitude de movimento, são medidas potencialmente úteis para trazermelhonias físicas, psicológicas e sociaise reduzir, portanto, aschancesde quedasnessapopulação.

De acordo com Boers etal., ${ }^{5}$ as quedas também são causadas porfatores ambientais, como pisos irregulares ou molhados, iluminação deficiente e escadas sem commãos. Esses fatores são susceptíveis a alterações, podendo ser usados em programas de prevenção das mesmas.

\section{CONCLUSÕES}

Concluiu-se que os déficits de equilíbrio e marcha são comuns entre idosos institucionalizados na cidade de Catanduva$\mathrm{SP}$, sendo mais freqüentemente encontrados entre os idosos mais velhos, do sexo feminino e naqueles com dificuldades visuais. Esses déficits contribuem para a ocorrência de quedas nessa população, e algumas me- 
didas de controle e prevenção de distúrbios de equilíbrio e marcha em idosos residentes em instituições asilares podeniam ser realizadas, destacando-se: o reconhecimento dos idosos com maiores dificuldades para se manter em postura ortostática e para caminhar, necessidade de exames penódicos para avaliar os estados de visão e audição e incentivo à prática de fisioterapia, visando ao fortalecimento musculare ganho de amplitude de movimento. $\mathrm{O}$ treinamento de equilíbrio e marcha são medidas potencialmente úteis para trazer melhonias físicas, psicológicas e sociais, além de reduzir as chances de quedas nessa população.

2. Freitas EV, Ghorayeb N, Pereira JBM, etal. Atividade física no idoso. In: Freitas EV, Py L, Neri AL, etal. Tratado de Geniatria e Gerontologia. Rio de Janeiro: Guanabara Koogan; 2002. p.857-65.

3. IBGE. 2000. Disponível em: URL: www.ibge.gov.br.

4. Papaléo Netto M. Epidemiologia do envelhecimento. In: .Gerontologia: a velhice e o envelhecimento em visão globalizada. São Paulo: Atheneu; 2002. p.26-43.

5. Boers I, Gerschlager W, Stalenhoef PA, Bloem BR. Falls in the elderly: II. Strategirs for prevention. Wien Klin Wochenschr 2001; 113: 398-407.

6. Rodrigues KL, Honda CM, Buriti MA. Problema da perda de equilíbrio na terceira idade: fatores de nisco e conseqüências. Revista fisio\&terapia 2004: 32-7.

7. Rosa TEC, Benício, MHD, Latorre, MRD O, Ramos, LR. Fatores

\section{NOTAS}

a Mestre em Ciências Médicas pela USP, docente da Faculdade de Filosofia, Ciências e Letras de Catanduva, SP.

E-mail: daniela_lojudice@hotmail.com

b Doutor em Medicina Preventiva, docente do Departamento de Medicina Social da Faculdade de Medicina da USP, Ribeirão Preto, SP.

E-mail: mlaprega@fmrp.usp.br

c,d Graduação em Fisioterapia pela Faculdade de Filosofia, Ciências e Letras de Catanduva, SP.

E-mails: prigardezani@yahoo.com.br; E-mail: privd@yahoo.com.br

\section{REFERÊNCIAS}

1. Chaimovicz F. A saúde dos idosos brasileiros às vésperas do século XXI: problemas, projeções e altemativas. Rev Saude Publica 1997; 31(2):148-200.

determinantes da capacidade funcional entre idosos. Rev Saude Publica 2003;37(1):40-8.

8. Tinetti ME. Penformance - oriented assessment of mobility problems in elderly patients. J Am Geriatr Soc 1986;34(2):119-26.

9. Baloh RW, Ying SH, Jacobson KM. A longitudinal study of gait and balance dysfunction in nomal older people. Arch Neurol 2003; 60: 835-9.

10. Steadman MCSP, Donaldson N, Kalra MD. A randomized controlled trial of an enhanced balance training program to improve mobility and reduce falls in eldenly patients. J Am Genatr Soc 2003; 51(6):847-52.

11. Ivers $R Q$, Boptom $M P H$, Cumming $R G$, Mitchell P, Freco F, Attebo K. Visual Impairment and falls in older adults: The blue mountains eye study. J Am Geriatr 
Soc 1998; 46 (1):58-64.

12. Pereira LSM, Basques FV, Marra TA. Avaliação da marcha em idosos. O Mundo da saúde 1999; 23(4): 221-8.

13. Tinetti ME, etal. A multifactorial intervention to reduce the nisk of falling among elderty people living in the community N EnglJ Med. 1994 Sep 29; 331(13): 821-7.

14. Whitney SL, Poole JL, Cass SP. A review of balance instruments for older adults. Am J Occup Ther 1998; 52(8):666-71.

15. Brasil. Ministério da Saúde. Conselho
Nacional de Saúde. Comissão Nacional de Ética em Pesquisa. Resolução 196/96 sobre pesquisa envolvendo seres humanos.

Brasília; 1996.

Recebido: 06/9/2007

Revisado: 08/4/2008

Aprovado: 30/4/2008 
